TUHEP-TH-02136

\title{
LMA Solution to the Solar Neutrino Problem and a Phenomenological Charged Lepton Mass Matrix
}

\author{
Xiao-Jun Bi \\ Department of Physics, Tsinghua University, \\ Beijing 100084, People's Republic of Chind \\ Yuan-Ben Dai \\ Institute of Theoretical Physics, Academia Sinica, \\ P.O. Box 2735, Beijing 100080, People's Republic of China it
}

(Dated: November 2, 2018)

\begin{abstract}
We propose a phenomenological form of the charged lepton mass matrix, which extends the idea of "lopsided" mass matrix in the literature. The features of the form are that both the 2-3 and 1-3 elements of the charged lepton mass matrix are of order 1 and that the small elements have a new structure. This form leads to an interesting result that both large atmospheric and solar neutrino mixing can be accounted for by the matrix. Another interesting result of this mass matrix is that it leads to very small 1-3 mixing in the lepton sector and can suppress the branching ratio of $\mu \rightarrow e \gamma$ under the present experimental limit in the supersymmetric see-saw case.
\end{abstract}

PACS numbers: $14.60 . \mathrm{Pq}$

*Email: bixj@mail.tsinghua.edu.cn

${ }^{\dagger}$ Email: dyb@itp.ac.cn 
The discovery of neutrino oscillation has been one of the most exciting experimental results in the last few years [1]. As the experimental data accumulating in the SuperKamiokande collaboration [2, [3] and more results published by SNO [4], K2K [5] and CHOOZ [6] experiments, the parameter space for the neutrino masses and mixing is narrowing down considerably. The recent analyses show that the atmospheric neutrino oscillation favors the $\nu_{\mu}-\nu_{\tau}$ process with a nearly maximal mixing angle $\sin ^{2} 2 \theta_{a t m} \geq 0.87$ and the mass squared difference $1.5 \times 10^{-3} \mathrm{eV}^{2} \leq \Delta m_{\text {atm }}^{2} \leq 4.8 \times 10^{-3} \mathrm{eV}^{2}$ at the $99 \%$ confidence level[7]. Among the four solutions for the solar neutrino deficits, the large mixing angle MSW (LMA) solution is most favored, followed by the LOW and VAC solutions [8]. The small mixing angle (SMA) solution is ruled out at the $2 \sigma$ level[3, [1]. The parameter space for the LMA solution is $0.2 \leq \tan ^{2} \theta_{\text {sol }} \leq 0.75$ and $2 \times 10^{-5} \mathrm{eV}^{2} \leq \Delta m_{\text {sol }}^{2} \leq 4 \times 10^{-4} \mathrm{eV}^{2}$ at the $3 \sigma$ confidence level, with the best fit values $\tan ^{2} \theta_{\text {sol }}=0.37$ and $\Delta m_{\text {sol }}^{2}=3.7 \times 10^{-5} \mathrm{eV}^{2}$,

On the theoretical side, hundreds of models for neutrino masses and mixings have been published during the last few years. However, a survey of these models shows that most of them yield the SMA or VAC solution for the solar neutrino problem [9], no matter whether the models produce the tiny neutrino masses directly at low energy or by the see-saw mechanism. In the following we will give a brief comment on the neutrino models in the literature and point out the difficulties in obtaining the LMA solution for the solar neutrino problem, and then present our model.

As we know, the neutrino mixing, which is described by the MNS matrix [10], is actually the mismatch between the two bases in which the mass matrix of the charged leptons or that of the active neutrinos is diagonal. The neutrino models are thus generally divided into two classes according to the origin of the large mixing angles: those from the charged lepton mass matrix $M_{L}$ or from the neutrino Majorana mass matrix $M_{\nu}$. For the models which generate the large mixing angles in $M_{\nu}$, it is usually difficult to reconcile the large mixing angles and a small mass squared splitting ratio $r \equiv \Delta m_{\text {sol }}^{2} / \Delta m_{\text {atm }}^{2} \approx 1.4 \times 10^{-2}$, which generally means a hierarchical structure of $M_{\nu}$. The LMA solution in this kind of models is usually achieved by fine tuning the parameters in non-see-saw case or by choosing a special hierarchy of the right handed neutrino spectrum in the see-saw case [9].

For models in which the large mixing angles coming from the charged lepton mass matrix the situation is better, since the large mixing and the small mass ratio come from different matrices. These models are either based on the beautiful idea of "flavor democracy" [1] or 
on the idea of "lopsided" form [12, 13] of the charged lepton mass matrix. However, having large mixing angles in $M_{L}$ raises the question why large CKM angles do not arise from the diagonalization of the quark mass matrices $M_{D}$ and $M_{U}$. Especially, in grand-unified models the Dirac mass matrices $M_{D}, M_{U}$ and $M_{L}$ are generally closely related.

The answer to this question in the "flavor democracy" models is that the CKM angles are small by a cancellation caused by an approximate symmetry between $M_{U}$ and $M_{D}$. This kind of models can predict a bi-maximal form of the MNS matrix by diagonalizing the charged lepton mass matrix and assuming the neutrino mass matrix diagonal at the same time. However, this kind of models tend to give a too large solar neutrino mixing angle near maximality, which is at most marginally consistent with the LMA parameter space.

The "lopsided" form of the charged lepton mass matrix is another elegant idea to give large lepton mixing while keeping small quark mixing at the same time. It is usually realized in the context of grand unified models, especially if an SU(5) symmetry plays a role in the form of the fermion mass matrices. In a SU(5) grand unified model, the left handed charged leptons are in the same multiplets as the $\mathrm{CP}$ conjugates of the right handed down quarks. Since the large mixing angle $\theta_{\text {atm }}$ is attributed to the mixing of the left-handed leptons in such models, it would generally be related by $S U(5)$ to a large mixing angle for the righthanded down quarks, which is not observable. On the other hand, the small CKM angles are related by $S U(5)$ to small mixings of the right-handed leptons, which are irrelevant to neutrino oscillation phenomena.

However, in most published models the "lopsided" form only accounts for the largeness of $\theta_{\text {atm }}$ and a SMA or VAC solution for the solar neutrino problem is usually predicted. To obtain a LMA solution in such models requires a right handed neutrino mass matrix with a complicate form and fine tuning of the parameters to some extent 12, 13.

One exception is given by K. S. Babu and S. M. Barr, where both the large solar and atmospheric neutrino mixing angles can be accounted for by diagonalizing the charged lepton mass matrix [14]. In their model, the charged lepton mass matrix has the following "lopsided" 
form ${ }^{1}$ :

$$
M_{L}=\left(\begin{array}{ccc}
0 & 0 & \rho^{\prime} \\
0 & \delta & \rho \\
\delta^{\prime} & \epsilon & 1
\end{array}\right) m_{D}
$$

with $\rho^{\prime} \sim \rho \sim 1, \delta \sim \delta^{\prime} \ll \epsilon \ll 1$. The parameters are determined by fitting the quark and lepton spectra and mixing angles. The unique nontrivial prediction of the model is $U_{e 3}$ element in the MNS matrix by diagonalizing $M_{L}$, which is $\left|U_{e 3}\right| \simeq 0.05$. This prediction will provide a test of this model.

However, most "lopsided" models predict a similar result of $U_{e 3} \simeq 0.05$. Thus a measurement of $U_{e 3} \simeq 0.05$ will not discriminate this model from others. Furthermore, the lepton flavor violating process $\mu \rightarrow e \gamma$ gives very strong constraints on the element $U_{e 3}$ [15, 16]. It is pointed out in [15] that the "lopsided" models, which always give a large $\mu-\tau$ mixing and a typical value of $U_{e 3} \simeq 0.05$, may lead to the branching ratio of $\mu \rightarrow e \gamma$ exceeding the present experimental upper limit in the supersymmetric see-saw case. This will be explained below. Actually, most "lopsided" models are realized in supersymmetric unified SO(10) models and the neutrino spectrum and mixing are given by see-saw mechanism. Thus, it seems that all these models meet the same difficulty in predicting the branching ratio of $\mu \rightarrow e \gamma$.

We have found a form of the charged lepton mass matrix independently when we tried to construct a "lopsided" model which can account for the LMA solution of the solar neutrino problem and simultaneously avoid the above difficulty on $\mu \rightarrow e \gamma$ decay. Our model is similar to the form (1) in the large elements in the third column but different in the structure of the first two columns. It gives a completely different prediction of $U_{e 3}$ and the branching ratio of $\mu \rightarrow e \gamma$ can be below the present experimental limit. In the following we shall first give our model and its features and then explain the smallness for $U_{e 3}$. In the last we shall discuss briefly its implication on the lepton flavor violating process $\mu \rightarrow e \gamma$ in supersymmetric see-saw case.

The aim of the work is to present a phenomenological model to account for the neutrino oscillation data and avoid the $\mu \rightarrow e \gamma$ difficulty. The mass matrices of the charged lepton

\footnotetext{
${ }^{1}$ Here we use the convention that a left-handed doublet multiplies the Yukawa coupling matrix from the left side while a right-handed singlet multiplies the matrix from the right side.
} 
and neutrino are assumed to have the following form:

$$
\begin{aligned}
M_{L} & =\left(\begin{array}{ccc}
0 & \delta & \sigma \\
-\delta & 0 & 1-\epsilon \\
0 & \epsilon & 1
\end{array}\right) m, \\
M_{\nu} & =\left(\begin{array}{ccc}
m_{1} & 0 & 0 \\
0 & m_{2} & 0 \\
0 & 0 & m_{3}
\end{array}\right),
\end{aligned}
$$

with $\sigma \sim \mathcal{O}(1), \epsilon \ll 1, \delta \ll \epsilon$. Similar to form (11) the model extends the usual "lopsided" form of the charged lepton mass matrix between the second and the third generations to include the first generation. For simplicity we have assumed that $M_{\nu}$ is diagonal here. The main feature of the model will not be changed if mixing in $M_{\nu}$ is small. Taking the values of the parameters

$$
\delta=0.00079, \epsilon=0.12, \text { and } \sigma=0.55
$$

we can obtain the correct mass ratios $m_{e} / m_{\mu}, m_{\mu} / m_{\tau}$ and the MNS matrix

$$
V_{M N S}=\left(\begin{array}{ccc}
0.851 & -0.525 & -0.0056 \\
0.362 & 0.595 & -0.718 \\
0.380 & 0.609 & 0.696
\end{array}\right) \text {. }
$$

This model then predicts the neutrino mixing parameters as

$$
\sin ^{2} 2 \theta_{\text {atm }}=0.999, \tan ^{2} \theta_{\text {sol }}=0.38 \text { and } U_{e 3}=-0.0056 \text {. }
$$

The notable feature of form (2) compared with the usual "lopsided" models is the order 1 element $\sigma$. We can see from Eq. (5) that by choosing a large $(2,3)$ and $(1,3)$ elements in $M_{L}$ we get two large mixing angles, which are corresponding to the maximal mixing of the atmospheric neutrinos and the large solar neutrino mixing in the LMA solution, by diagonalizing the charged lepton mass matrix. In this phenomenological model we separate the large angles $\theta_{\text {atm }}$ and $\theta_{\text {sol }}$ from the small mass ratio $\Delta m_{\text {sol }}^{2} / \Delta m_{\text {atm }}^{2}$ completely. Thus it is very easy to reconcile the large mixing angles and the neutrino spectrum of the LMA solution without any fine tuning.

Unlike the "flavor democracy" models, which usually predict a bi-maximal MNS matrix, our model gives the best fit value of $\theta_{\text {sol }}$ for the LMA solution. It is unlike the usual 
"lopsided" models either, which predict a small lepton mixing angle between the first and the second generations with a typical value of $\sqrt{m_{e} / m_{\mu}} \approx 0.07$ [12, 13]. This phenomenological form may provide a new possibility of model-building for the fermion masses and mixing.

Besides $\theta_{\text {atm }} \approx \pi / 4$ the prediction of $U_{e 3}=-0.0056$ is quite non-trivial, since all the parameters are fixed by the lepton mass ratios and the solar neutrino mixing angle. This prediction is different from most other "lopsided" models, including that given in Ref. [14]. It thus provides a test of our model.

The smallness of $U_{e 3}$ can be explained as following. The charged lepton mass matrix $M_{L}$ can be diagonalized by a bi-unitary rotation

$$
U_{L}^{\dagger} M_{L} U_{R}=\left(\begin{array}{lll}
m_{e} & & \\
& m_{\mu} & \\
& & m_{\tau}
\end{array}\right) .
$$

The MNS matrix is defined by $U_{M N S}=U_{L}^{\dagger} U_{\nu}$, where $U_{\nu}$ is the unitary matrix diagonalizing the neutrino mass matrix and $U_{\nu}=I$ here. Thus $U_{M N S}$ is given by $U_{L} . U_{L}$ can be approximately obtained by rotating the left-handed charged leptons so that the right-upper triangle of $M_{L}$ in (2) becomes zeros. This can be achieved by three steps. First we rotate the first two generation charged leptons by a matrix

$$
V_{12}=\left(\begin{array}{ccc}
c_{12} & s_{12} & 0 \\
-s_{12} & c_{12} & 0 \\
0 & 0 & 1
\end{array}\right),
$$

with $c_{12}=\cos \theta_{12}, s_{12}=\sin \theta_{12}$ and $\tan \theta_{12}=\sigma /(1-\epsilon)$. Then the $1-3$ element of $M_{L}$ becomes zero and the $2-3$ element becomes $\sigma^{\prime}=\sqrt{\sigma^{2}+(1-\epsilon)^{2}}$. The second step is a similar rotation between the second and the third generations, $V_{23}$, with $\tan \theta_{23}=\sigma^{\prime}$, which makes the $2-3$ element of $M_{L}$ zero. The final step is a small rotation $V_{12}^{\prime}$ which makes the $1-2$ element zero. The rotation angle is $\theta_{12}^{\prime} \cong-\frac{c_{12} \delta}{s_{23}}$. We then get the unitary matrix

$$
U_{L}^{\dagger}=V_{12}^{\prime \dagger} V_{23}^{\dagger} V_{12}^{\dagger}
$$

from which we have that $\theta_{\text {atm }} \cong-\theta_{23}, \theta_{\text {sol }} \cong-\theta_{12}$ and $U_{e 3} \cong \theta_{12}^{\prime} \sin \theta_{23}$. Using the expressions of the diagonal elements of the resulting triangular matrix $U_{e 3}$ can be approximately expressed by mass ratio and physical mixing angles as

$$
U_{e 3} \cong \frac{m_{e}}{m_{\mu}} \cdot U_{\mu 3} / \tan \theta_{\text {sol }} .
$$


On the other hand, the $U_{e 3}$ from (1) can be expressed by

$$
U_{e 3} \cong-\sqrt{\frac{m_{e}}{m_{\mu}}} \cdot U_{\mu 3} .
$$

From the Eqs. (10) and (11) we can see that, for large $\theta_{s o l}, U_{e 3}$ in our model is suppressed compared to that in Ref. [14] due to the smallness of $m_{e} / m_{\mu}$. The difference in the dependence of $U_{e 3}$ on the mass ratio of $m_{e} / m_{\mu}$ originates from the different structures in $M_{L}$ in these two models.

A $U_{e 3} \sim 0.05$ is at the edge of the parameter space measurable in the next generation long baseline neutrino experiments, which may improve the present sensitivity to $\sin ^{2} 2 \theta_{13}$ by an order of magnitude, probing $\sin ^{2} 2 \theta_{13}$ at the level of $0.01[17]$. To discriminate the two kinds of models, an entry-level neutrino factory is necessary, which can probe $\sin ^{2} 2 \theta_{13}$ down to $10^{-3}$ [18]. If no $\nu_{\mu}-\nu_{e}$ signal is observed even at this level, most of the "lopsided" models may then be ruled out. If $U_{e 3}$ is indeed as small as that in our model, the high-performance neutrino factory, which can probe $\sin ^{2} 2 \theta_{13}$ down to the order of $10^{-4}$, will be necessary to measure its value 19.

As for the quark sector, we will not discuss it in detail here. We only want to point out that a large $(1,3)$ element in $M_{L}$ will not lead to a large quark mixing if the relation $M_{D} \sim M_{L}^{T}$ is satisfied. Unlike the large elements in the mass matrices, the relations between the small matrix elements in the quark sector and those in the lepton sector depend on the details of the grand-unified models used. The simplest possible form of the down quark mass matrix is obtained by first adding a small $(3,1)$ element and a coefficient $-\frac{1}{3}$ in front of $\epsilon$ in $M_{L}$, and then transposing it. Then $M_{D}$ can produce acceptable down quark spectrum and mixing angles.

Finally, we discuss the case that the neutrino mass matrix is generated by the see-saw mechanism,

$$
M_{\nu}=-M_{N} M_{R}^{-1} M_{N}^{T}
$$

where $M_{N}$ is the neutrino Dirac mass matrix. In this case we can modify our assumption to that the charged lepton mass matrix of the form in Eq. (22) is given in the basis where the Dirac neutrino mass matrix $M_{N}$ is diagonal. Generally the right-handed Majorana mass matrix $M_{R}$ may be non-diagonal. Then $M_{\nu}$ in Eq. (12) is not diagonal and deviates from Eq. (3). If mixing in $M_{R}$ is small, this deviation is small and the main feature of our model described above remains intact. Another possibility is that $M_{N}$ is hierarchical and 
this structure transfer to $M_{\nu}$ through the see-saw mechanism[9]. Taking hierarchy of $M_{N}$ similar to that of up quarks as suggested in $\mathrm{SO}(10)$ grand unified models and assuming no large hierarchy among the elements of $M_{R}^{-1}$, we find that the mixing in $M_{\nu}$ is tiny, which almost do not change the values of $V_{M N S}$ in Eq. (5).

The main virtue of the assumption that $M_{L}$ has the form (2) in the basis where $M_{N}$ is diagonal is that it can avoid the contradiction with the $\mu \rightarrow e \gamma$ experiments when the "lopsided" form is realized in the supersymmetric see-saw case. In this case and if supersymmetry breaking is mediated by gravity the soft SUSY breaking terms will introduce new lepton flavor violating sources. The mechanism is that there are two Yukawa coupling matrices $Y_{L}$ and $Y_{N}$ in the lepton sector for the charged leptons and neutrinos at the energy scale above the right-handed neutrino masses. They are proportional to $M_{L}$ and $M_{N}$ respectively. These two Yukawa coupling matrices can not be diagonalized simultaneously and will lead to lepton flavor mixing, in analogy to the flavor mixing in the quark sector. This lepton flavor mixing can transfer to the scalar lepton sector through quantum effects. The masses of sleptons are usually assumed to be universal at the SUSY breaking scale to avoid the low energy flavor problems. The flavor mixing effects in the scalar lepton sector in the low energy region can then be given approximately by

$$
\left(\delta m_{\tilde{L}}^{2}\right)_{i j} \approx \frac{1}{8 \pi^{2}} V_{i 3} V_{j 3}^{*} \cdot Y_{N_{3}}^{2}\left(3+a^{2}\right) m_{0}^{2} \log \frac{M_{G U T}}{M_{R}},
$$

where $\left(\delta m_{\tilde{L}}^{2}\right)_{i j}$ is the non-diagonal terms of the scalar lepton mass matrix, representing the lepton flavor mixing between the $i$-th and the $j$-th generations. $Y_{N_{3}}$ is the third generation Yukawa coupling of neutrinos, which dominates over the first two generations. $V$ is the mixing matrix in the lepton sector. The loop effect with internal SUSY particles is much more effective than that with internal neutrinos in producing the $\mu \rightarrow e \gamma$ process so that the rate of this process is determined by $\left(\delta m_{\tilde{L}}^{2}\right)_{12}$, which is proportional to $V_{23} V_{13}^{*}$. Since there is a very high experimental sensitivity to the process $\mu \rightarrow e \gamma\left(\operatorname{Br}(\mu \rightarrow e \gamma)<1.2 \times 10^{-11}\right.$ [21]), and $V_{23}$ is large (around $1 / \sqrt{2}$ ) in "lopsided" models, $V_{13}$ is thus very strongly constrained.

In the usual "lopsided" models, with the typical value of $U_{e 3} \sim 0.05$, the branching ratio of $\mu \rightarrow e \gamma$ is then given by 15

$$
\operatorname{Br}(\mu \rightarrow e \gamma) \approx C \cdot 10^{-7}\left(\frac{100 G e V}{m_{s}}\right)^{4}\left(\frac{\tan \beta}{10}\right)^{2},
$$

where $C$ is around $1 \sim 10$. Thus, if the common SUSY particle mass scale, $m_{s}$, is $\lesssim 1 T e V$ and $\tan \beta$ is not too small, then the branching ratio should be above the present experimental 
limit. This fact has been noticed by a few authors [15, 16]. However, in our model, by the assumption that $M_{L}$ has the form (2) in the basis where $M_{N}$ is diagonal, $V_{13}$ can be as small as -0.0056 . Since the rate for the process $\mu \rightarrow e \gamma$ is approximately proportional to $V_{13}^{2}$ it is greatly suppressed compared to it's value in the usual "lopsided" models. Our model predicts a branching ratio of $\mu \rightarrow e \gamma$ not too much smaller than the present experimental limits and easily to be detected in the future experiments[22]. This is an important difference of our model from the model of Ref. [14. Like the usual "lopsided" models, Ref. [14 predicts a $\mu \rightarrow$ e $\gamma$ branching ratio already exceeding the present experimental limit.

The process $\tau \rightarrow \mu \gamma$ is another promising process to determine whether there is a large mixing between the second and the third generations in the charged lepton sector, since its rate is proportional to $\left|V_{23}^{*} V_{33}\right|^{2}$. If both processes are found in the future experiments in the range of $10^{-6}>\operatorname{Br}(\tau \rightarrow \mu \gamma)>10^{-9}$ and $10^{-11}>\operatorname{Br}(\mu \rightarrow e \gamma)>10^{-14}$ [21, 22], our model, with a large $2-3$ mixing while very small $1-3$ mixing, will be a very attractive candidate to accommodate the observations.

In summary, we have constructed a phenomenological form of the charged lepton mass matrix, which provides a new possibility in model construction. This form can produce the large solar neutrino mixing angle and the maximal atmospheric neutrino mixing angle simultaneously without any fine tuning. With such a charged lepton mass matrix it is very easy to build a neutrino model to explain the observed neutrino oscillation experiments. The unique prediction of this model is that $U_{e 3}=-0.0056$, which is different from predictions of all other "lopsided" models. This prediction can be tested in neutrino factory. This model has another virtue that it predicts the branching ratio of $\mu \rightarrow e \gamma$ below the present experimental limit but may be not too much smaller than it in the SUSY-GUT see-saw case. If both the $\tau \rightarrow \mu \gamma$ and $\mu \rightarrow e \gamma$ processes are detected in the near future experiments, our model will be an attractive candidate to explain the experimental results. 


\section{Acknowledgments}

This work is supported by the National Natural Science Foundation of China under the grand No. 10105004 and No. 19835060.

[1] Y. Fukuda et al., Super-Kamiokande Collaboration, Phys. Rev. Lett. 811562 (1998); 822644 (1999); 821810 (1999); 822430 (1999).

[2] Y. Fukuda et al., Super-Kamiokande Collaboration, Y. Fukuda et al., Super-Kamiokande Collaboration, Phys. Rev. Lett. 865651 (2001).

[3] Y. Fukuda et al., Super-Kamiokande Collaboration, Phys. Rev. Lett. 865656.

[4] Q. R. Ahmad et al., SNO Collaboration, Phys. Rev. Lett. 87, 071301 (2001).

[5] S. H. Ahn et al., K2K Collaboration, Phys. Lett. B511 178 (2001).

[6] M. Apollonio et al., CHOOZ Collaboration, Phys. Lett. B420 397 (1998); B466 415 (1999).

[7] T. Toshito, Super-Kamiokande Collaboration, hep-ex/0105023; G. I. Fogli, E. Lisi and A. Marrone, hep-ph/0110089.

[8] J. N. Bahcall, P. I. Krastev and A. Yu. Smirnov, JHEP 0105, 015 (2001); J. N. Bahcall, M. C. Gonzalez-Garcia and C. Pena-Garay, JHEP 0108, 014(2001); J. N. Bahcall, M. C. Gonzalez-Garcia and C. Pena-Garay, hep-ph/0111150.

[9] S. M. Barr and Ilja Dorsner, Nucl. Phys. B585, 79 (2000); Ilja Dorsner and S. M. Barr, Nucl. Phys. B617, 493 (2001) and references therein.

[10] Z. Maki, M. Nakagawa and S. Sakata, Prog. Theor. Phys. 28870 (1962).

[11] H. Fritzsch and Z.Z. Xing, Phys. Lett. B372, 265 (1996); Phys. Lett. B440, 313 (1998); Prog. Part. Nucl. Phys. 45, 1 (2000); M. Fukugita, M. Tanimoto and T. Yanagida, Phys. Rev. D 57, 4429 (1998); Phys. Rev. D 59, 113016 (1999); M. Tanimoto, Phys. Rev. D59, 017304 (1999); R. Mohapatra and S. Nussinov, Phys. Lett. B441, 299 (1998); S.K. Kang and C.S. Kim, Phys. Rev. D 59, 091302 (1999).

[12] C. H. Albright and S.M. Barr, Phys. Rev. D 64, 073010 (2001); C. H. Albright and S.M. Barr, Phys. Rev. D 62, 093008 (2000); C. H. Albright, S. Geer, hep-ph/0108070.

[13] K. S. Babu, Jogesh C. Pati, Frank Wilczek, Nucl. Phys. B566, 33 (2000); G. Altarelli and F. Feruglio, JHEP 9811, 021 (1998); G. Altarelli and F. Feruglio, Phys. Lett. B451, 388 (1999); 
K. Hagiwara and N. Okamura, Nucl. Phys. B548, 60 (1999); N. Irges, S. Lavignac and P. Ramond, Phys. Rev. D 58, 035003 (1998); W. Buchmüller and T. Yanagida, Phys. Lett. B445, 399 (1998); M. Bando and T. Kugo, Prog. Theor. Phys. 101, 1313 (1999); M. Bando, T. Kugo, and K. Yoshioka, Prog. Theor. Phys. 104, 211 (2000); J. Sato and T. Yanagida, Phys. Lett. B430, 127 (1998); J. Sato and T. Yanagida, Phys. Lett. B493 356 (2000); Y. Nomura and T. Yanagida, Phys. Rev. D 59, 017303 (1999); N. Haba, Phys. Rev. D 59, 035011 (1999); Z. Berezhiani and A. Rossi, JHEP 9903, 002 (1999); K. Izawa, K. Kurosawa, N. Nomura, and T. Yanagida, Phys. Rev. D 60, 115016 (1999); P. Frampton and A. Rasin, Phys. Lett. B478, 424 (2000); R. Barbieri, G. Kane, L.J. Hall, and G.G. Ross, hep-ph/9901228;R. Kitano and Y. Mimura, Phys. Rev. D 63, 016008 (2001).

[14] K. S. Babu and S. M. Barr, Phys. Lett. B525, 289 (2002).

[15] X. J. Bi and Y. B. Dai, hep-ph/0112375 to appear in Phys. Rev. D.

[16] J. Sato, K. Tobe, T. Yanagida, Phys. Lett. B498 189(2001); J. Sato and K. Tobe, Phys. Rev. D 63116010 (2001).

[17] See, for example, MINOS Collaboration, NuMI-L-375 (1998).

[18] V. Barger, S. Geer, R. Raja, K. Whisnant, Phys. Rev. D62 073002(2000).

[19] S. Geer and H. Schellman "Physics at a Neutrino Factory", Report to the Fermilab Directorate, FERMILAB-FN-692, April 2000; S. Geer, FERMILAB-Pub-00/195-T, hep-ph/0008155.

[20] G. Altarelli and F. Feruglio, JHEP 11, 21 (1998); G. Altarelli and F. Feruglio, Phys. Rept. 320, 295 (1999).

[21] M. L. Brooks et al., MEGA Collaboration, Phys. Rev. Lett. 831521 (1999); S. Ahmed et al., CLEO Collaboration, Phys. Rev. D 61071101 (2000); U. Bellgardt et al., Nucl. Phys. B229 1 (1988).

[22] L. M. Barkov et al., Research Proposal for an experiment at PSI (1999); M. Bachmann et al., MECO collaboration, Research Proposal E940 for an experiment at BNL (1997). 